Research article

Open Access

\title{
A comprehensive analysis of prognostic signatures reveals the high predictive capacity of the Proliferation, Immune response and RNA splicing modules in breast cancer
}

\author{
Fabien Reyal1,2,3, Martin H van Vliet ${ }^{4,5}$, Nicola J Armstrong ${ }^{4}$, Hugo M Horlings ${ }^{1}$, Karin E de Visser 6 , \\ Marlen Kok1, Andrew E Teschendorff7, Stella Mook1, Laura van 't Veer'1, Carlos Caldas?, \\ Remy J Salmon ${ }^{3}$, Marc J van de Vijver ${ }^{1,8}$ and Lodewyk FA Wessels ${ }^{4,5}$
}

\author{
1Department of Pathology, The Netherlands Cancer Institute, Plesmanlaan 121, 1066 CX Amsterdam, The Netherlands \\ 2Department of Surgery, Institut Curie, 6 rue d'Ulm, 75005 Paris, France \\ 3UMR 144, CNRS-Institut Curie, Molecular Oncology Team, 12 rue Lhomond, 75005 Paris, France \\ ${ }^{4}$ Bioinformatics and Statistics Group, The Netherlands Cancer Institute, Plesmanlaan 121, 1066 CX Amsterdam, The Netherlands \\ ${ }^{5}$ Faculty of EEMCS, Delft University of Technology, Mekelweg 4, 2628 CD Delft, The Netherlands \\ ${ }^{6}$ Department of Molecular Biology, The Netherlands Cancer Institute, Plesmanlaan 121, 1066 CX Amsterdam, The Netherlands \\ ${ }^{7}$ Cancer Research UK, Cambridge Research Institute and Department of Oncology, Li Ka Shing Centre, University of Cambridge, Cambridge CB2 \\ ORE, UK \\ 8Department of Pathology, Academic Medical Center, Meibergdreef 9, 1100 DD Amsterdam The Netherlands
}

Corresponding author: Lodewyk FA Wessels, l.wessels@nki.nl

Received: 29 Mar 2008 Revisions requested: 1 May 2008 Revisions received: 31 Jul 2008 Accepted: 13 Nov 2008 Published: 13 Nov 2008

Breast Cancer Research 2008, 10:R93 (doi:10.1186/bcr2192)

This article is online at: http://breast-cancer-research.com/content/10/6/R93

(C) 2008 Reyal et al.; licensee BioMed Central Ltd.

This is an open access article distributed under the terms of the Creative Commons Attribution License (http://creativecommons.org/licenses/by/2.0), which permits unrestricted use, distribution, and reproduction in any medium, provided the original work is properly cited.

\begin{abstract}
Introduction Several gene expression signatures have been proposed and demonstrated to be predictive of outcome in breast cancer. In the present article we address the following issues: Do these signatures perform similarly? Are there (common) molecular processes reported by these signatures? Can better prognostic predictors be constructed based on these identified molecular processes?

Methods We performed a comprehensive analysis of the performance of nine gene expression signatures on seven different breast cancer datasets. To better characterize the functional processes associated with these signatures, we enlarged each signature by including all probes with a significant correlation to at least one of the genes in the original signature. The enrichment of functional groups was assessed using four ontology databases.
\end{abstract}

Results The classification performance of the nine gene expression signatures is very similar in terms of assigning a sample to either a poor outcome group or a good outcome group. Nevertheless the concordance in classification at the sample level is low, with only $50 \%$ of the breast cancer samples classified in the same outcome group by all classifiers. The predictive accuracy decreases with the number of poor outcome assignments given to a sample. The best classification performance was obtained for the group of patients with only good outcome assignments. Enrichment analysis of the enlarged signatures revealed 11 functional modules with prognostic ability. The combination of the RNA-splicing and immune modules resulted in a classifier with high prognostic performance on an independent validation set.

Conclusions The study revealed that the nine signatures perform similarly but exhibit a large degree of discordance in prognostic group assignment. Functional analyses indicate that proliferation is a common cellular process, but that other functional categories are also enriched and show independent prognostic ability. We provide new evidence of the potentially promising prognostic impact of immunity and RNA-splicing processes in breast cancer.

\section{Introduction}

Breast cancer is composed of distinct diseases with different outcomes. Clinical and pathological factors are currently employed to determine the prognosis of patients. The Saint- 
Gallen guidelines [1], National Institute of Health guidelines [2] and Nottingham Prognostic Index guidelines [3] as well as the AdjuvantOnline! decision-making tools [4] use a combination of these prognostic factors to adapt adjuvant treatment based on the prognosis prediction. Owing to insufficiently accurate prognosis predictions, however, a substantial proportion of breast cancer patients with breast cancer of inherently good outcome receive adjuvant systemic therapy without gaining any benefit [5].

High-throughput technologies such as gene expression microarrays can offer new opportunities to improve the ability to determine individual prognosis. After confirmation of their performance in validation studies, classifiers such as the 70-gene signature developed at the Netherlands Cancer Institute $[6,7]$ and the 76-gene signature developed at the Erasmus Medical Center $[8,9]$ may become of use in clinical practice. Similarly, Paik and colleagues built a 16-gene classifier for paraffinembedded tissues (OncotypeDX ${ }^{\odot}$; Genomic Health, Redwood, California, USA) using quantitative RT-PCR [10]. The ongoing MINDACT trial [11] and TAILORx trial [12] aim to confirm the performance of the 70-gene and the OncotypeDX signatures on large populations of 6,000 patients and 10,000 patients, respectively. In the meantime, it has become commonplace for research groups to define new classifiers with a potentially higher level of performance than existing classifiers [13-21].

Publications have raised several concerns about microarray studies that would potentially impair the use of gene expression classifiers in clinical routine [22-24]. We performed a comprehensive analysis of gene-expression-based classifiers on a collection of 1,127 breast cancer samples all hybridized on the Affymetrix ${ }^{\odot}$ platform. To the best of our knowledge, this represents the largest, single platform dataset employed to evaluate prognostic classifiers to date.

Nine gene expression signatures derived using diverse methodological approaches and focusing on various aspects thought to be associated with breast cancer outcome were selected: the 76-gene signature employed to compute the Relapse score [8]; the 52-gene and 17-gene signatures employed to compute the Molecular Prognostic Index (T52 and T17) [20]; the Intrinsic/UNC gene set used to define the molecular subtypes [15]; the 70-gene and 25-gene Chromosomal Instability signatures (CIN70 and CIN25) [13]; the Core Serum Response signature [14]; the Invasiveness Gene Signature [16]; and the 97-gene signature used to derive the Gene expression Grade Index [25]. (Note that there are nine signatures in total, but T17 and CIN25 are smaller versions of T52 and CIN70, respectively. T17 and CIN25 were therefore not included in the enlargement and enrichment analyses.) Two well-known signatures, the 21-gene Genomic Health signature [10] and the 70-gene signature from the Netherlands Cancer Institute $[6,7]$, were not included. The Genomic Health signature was not included since it is not a micro-array-based gene expression signature but is designed for RT-PCR assays. The 70-gene signature was not included since the 295 sample dataset on which this signature was developed was employed as a completely independent validation set. Throughout the text we use signature to refer to both the gene set as well as the classifier, which, based on this gene set, assigns a sample to an outcome group.

While some signatures were designed for specific subgroups of patients, we applied the signatures to the complete, unstratified set of breast cancer samples. We followed this approach for several reasons. First, a large dataset maximizes power to detect common prognostic factors, while stratification into subtypes results in smaller groups and, therefore, in reduced power. Second, there is mounting evidence that classifiers designed within a specific subgroup also perform well in other subgroups. For example, the 70-gene classifier developed by van 't Veer and colleagues was developed for lymph-nodenegative patients [6], but performs very well on lymph-nodepositive patients as demonstrated by van de Vijver and colleagues [7]. In fact, patients with up to three positive lymph nodes are now included in the MINDACT trial [26]. Similarly, the 21-gene OncotypeDX signature, which was developed for node-negative disease, has also been shown to be a good predictor of disease outcome in node-positive patients [27]. These facts indicate that breast cancer subgroups as defined by classical markers are currently being re-evaluated, and that the signatures have some common core of biological/molecular processes involved in the development of breast cancer that is critical for predicting good outcome or poor outcome. Finally, an analysis performed on the complete dataset provides a benchmark against which subtype specific signature performance can be gauged and also reveals whether predictors designed on the whole cohort have any predictive value in subgroups.

We addressed the following issues: Do these nine separate signatures perform similarly? Are there (common) molecular processes reported by these signatures? Can better prognostic predictors be constructed based on (a combination of) these identified molecular processes?

\section{Materials and methods}

Figure 1 depicts a graphical overview of the analysis procedure followed. A detailed Materials and methods section is available in Additional data file 1.

\section{Data preprocessing}

Six breast cancer datasets $[9,17-19,28,29]$ - all arrayed on the same platform (HGU-133A Affymetrix ${ }^{\odot}$ Santa Clara, California, USA) to avoid cross-platform discrepancies - for which the raw data (.CEL files) were publicly available were downloaded from the Gene Expression Omnibus and ArrayExpress repository websites $[30,31]$. This resulted in a total of 1143 


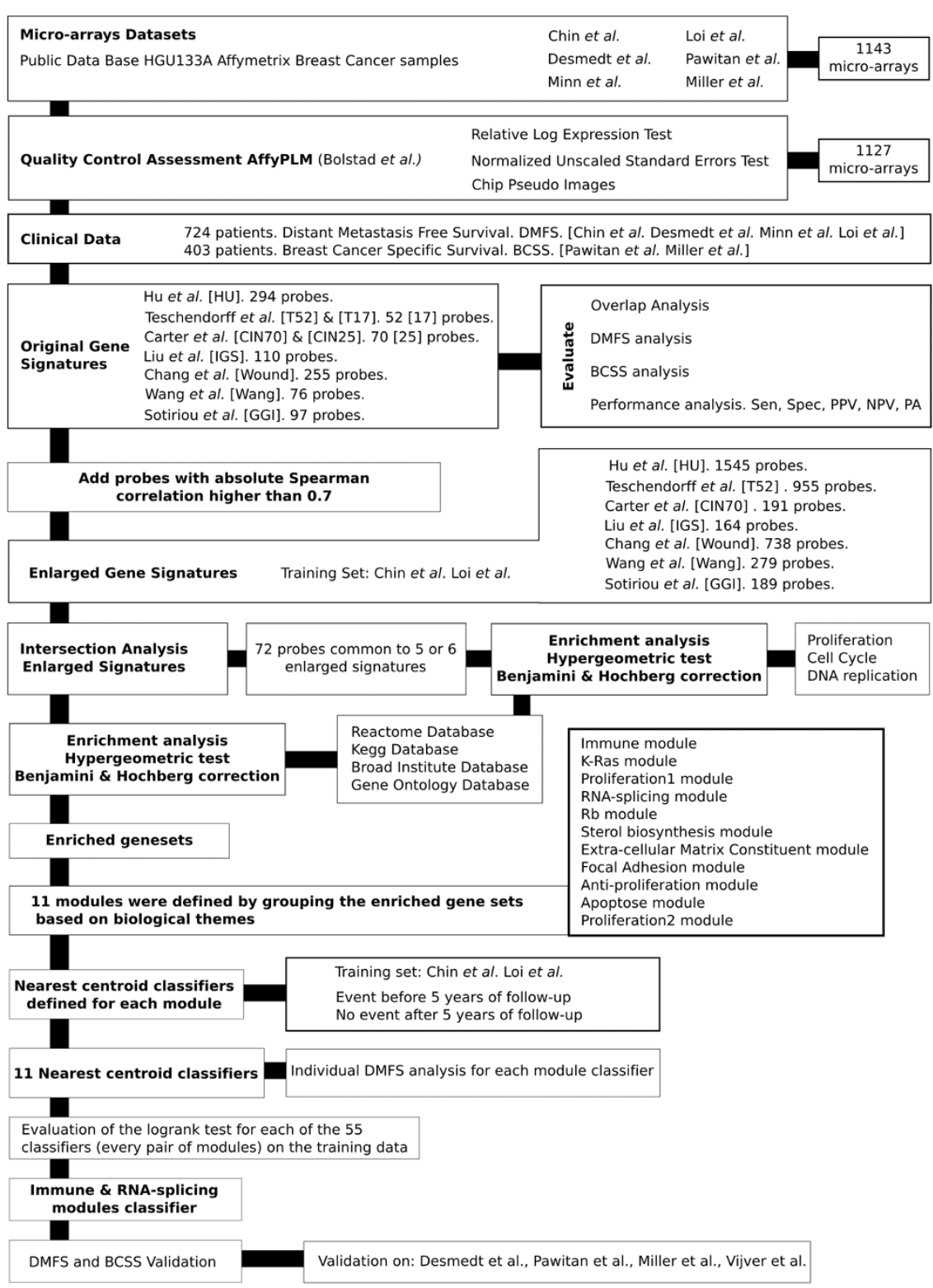

Detailed overview of the datasets, gene sets and analysis steps employed. PPV, positive predictive value; NPV, negative predictive value; PA, predictive accuracy.

microarrays. Of these, 1,127 were deemed to be of sufficient quality and were kept in the current study. Sixteen micro-arrays were rejected due to major artifacts in the original .CEL files.

To ensure comparability between the different datasets, they were all subjected to the same preprocessing procedure. Microarray quality-control assessment was carried out using the AffyPLM R-package [32]. Selected arrays were normalized using the RMA expression measure algorithm [33]. For 724 samples, distant metastasis-free survival (DMFS) data were available; while for 403 samples, breast cancer-specific survival (BCSS) data were available. For some samples both
DMFS and BCSS information is available. For the performance analyses, where the results per endpoint are reported separately, the overlapping samples were analyzed twice (once for each endpoint).

For the enlargement of the signatures and the construction of the functional classifiers, only the Chin and Loi datasets were used - resulting in a total of 450 samples. We will refer to this combined dataset as the Chin-Loi training set. Clinical and histopathological features for all samples are summarized in Table 1. 
Table 1

Clinical and histopathological features of 1,127 patients presenting breast carcinoma and included in the survival analyses

\begin{tabular}{|c|c|c|}
\hline & Distant metastasis-free survival & Breast cancer-specific survival \\
\hline Patients (n) & 724 & 403 \\
\hline \multicolumn{3}{|l|}{ Tumor size } \\
\hline Number $(\%)$ of $\mathrm{T} 1(\leq 20 \mathrm{~mm})$ & $292(43 \%)$ & $271(69 \%)$ \\
\hline Mean (standard deviation) size (mm) & $25.3(13)$ & $22.2(10.4)$ \\
\hline \multicolumn{3}{|l|}{ Histological grading: Elston Ellis } \\
\hline Grade I & $104(15.4 \%)$ & $88(23 \%)$ \\
\hline Grade II & $253(37.5 \%)$ & $176(45 \%)$ \\
\hline Grade III & $195(28.9 \%)$ & $111(28 \%)$ \\
\hline Not available & $121(17.9 \%)$ & $13(3 \%)$ \\
\hline \multicolumn{3}{|l|}{ Genomic grade index } \\
\hline High grade & $331(49 \%)$ & $179(46 \%)$ \\
\hline \multicolumn{3}{|l|}{ Estrogen receptor status: immunohistochemistry } \\
\hline Positive & $488(72.5 \%)$ & $197(51 \%)$ \\
\hline Not available & $6(0.8 \%)$ & $160(40 \%)$ \\
\hline \multicolumn{3}{|l|}{ Estrogen receptor status: gene expression } \\
\hline Positive & $496(74 \%)$ & $297(76 \%)$ \\
\hline \multicolumn{3}{|l|}{ Her2 status: gene expression } \\
\hline Positive & $86(13 \%)$ & $50(13 \%)$ \\
\hline \multicolumn{3}{|l|}{ Lymph node metastasis } \\
\hline Positive & $197(28 \%)$ & $76(20 \%)$ \\
\hline Not available & $8(1 \%)$ & $165(41 \%)$ \\
\hline \multicolumn{3}{|l|}{ Distant metastasis or death from breast cancer } \\
\hline Positive & $177(26 \%)$ & $83(21 \%)$ \\
\hline Within 5 years & $131(19 \%)$ & $59(15 \%)$ \\
\hline Mean (standard deviation) follow-up (months) & $85.6(53.4)$ & $89.6(39.8)$ \\
\hline Datasets used (Ref) & {$[9,17,19,29]$} & {$[18,28]$} \\
\hline
\end{tabular}

Sixty-six patients out of 1,127 samples were excluded from the survival analysis due to missing information (time or event censoring).

\section{Signature validation}

To the complete dataset of 1,127 samples, we applied the 76gene signature [8], the Intrinsic/UNC signature [15], the Chromosomal Instability signatures (CIN70 and CIN25) [13], the Core Serum Response signature [14], the Invasiveness Gene Signature [16], the Molecular Prognosis Index signatures (T52 and T17) [20], and the Gene expression Grade Index signature [25]. Survival analyses were performed using the KaplanMeier estimate of the survival function. Comparison between survival curves was performed using the log-rank test. Hazard ratios were estimated using the Cox proportional hazard model. $P$ values were considered significant when $<0.05$. Only variables with a significant $P$ value in univariate analyses were included in a multivariate model. Time-censoring analyses were performed using right censoring of the events from 1 to 12 years. The performance analysis (sensitivity, specificity, positive predictive value, negative predictive value, and predictive accuracy) of the signatures was carried out using the ROCR package [32]. For these analyses, the outcome was dichotomized into a poor outcome group (samples with an event before 5 years of follow-up) and a good outcome group (samples with no event and a follow-up of at least 5 years). The analyses were performed using $\mathrm{R}$ software [32] and Matlab software [34].

\section{Enlarged signatures}

We created enlarged signatures by including all probes with an absolute Spearman rank order correlation $>0.7$ with at least one of the genes in the original signatures. This calculation was performed on the Chin-Loi training set. 


\section{Enrichment analyses of signatures}

For all signatures, we evaluated whether specific gene sets (that is, functional groups), are overrepresented. We gathered a collection of 5,480 gene sets from four databases: Gene Ontology [35], Kyoto Encyclopedia of Genes and Genomes [36], Reactome [37] and the Molecular Signatures Database [38] (see Materials and methods in Additional data file 1). The hypergeometric test was employed to test the significance of the overlap between each signature and gene set. Multiple testing adjustment was performed by applying the BenjaminiHochberg correction (per signature) [39].

\section{Discovery and validation of module classifiers}

The gene sets that were enriched in at least one signature were grouped into modules based on common functional annotation. The only exception was the group of proliferationrelated gene sets. This group was split into two groups, the first containing gene sets common to either five or six enlarged signatures while the second contained the remaining proliferation gene sets. This resulted in 11 functional modules. For each of these modules we constructed a nearest mean classifier using the genes common between the enlarged signatures and the group of genes in the module. The classifier was trained using the Chin-Loi training set. For each module, the poor outcome centroid was derived from samples with a metastatic event before 5 years of follow-up; the good outcome centroid was derived from samples with no metastatic events and a follow-up longer than 5 years. Based on the genes associated with a given module, the Spearman rank correlation between each sample and the centroid values of the poor outcome and the good outcome centroids were determined. Each sample in the training set was then assigned to the centroid with which it showed the highest correlation (the nearest centroid).

We then created classifiers by combining the output of every pair of module classifiers. For each pair of modules, this results in a three-class classifier: samples with two poor (good) group labels are assigned to the poor (good) group, while samples with discordant labels are assignment to the intermediate group. The performance of these classifiers was evaluated, based on the log-rank test, on the time-to-event data on the Chin-Loi training set. The best module-pair classifier was selected to enter the validation stage.

We validated the best module-pair classifier on independent data. Validation was performed for DMFS on the datasets of Desmedt and colleagues [9] and Minn and colleagues [19], while for BCSS the datasets of Pawitan and colleagues [28] and Miller and colleagues [18] were used . In addition, we validated the best module-pair classifier on the dataset of van de Vijver and colleagues [7]. The gain in predictive accuracy of the classifier, as compared with common clinical staging systems, was investigated on the dataset of van de Vijver and colleagues using the method of Schemper and Henderson [40], implemented in the R package software [41] . Dunkler and colleagues have also recently used this approach in the context of gene signatures [42]. Briefly, predictive inaccuracy is calculated as the average of the absolute difference between observed outcomes and the model predictions. The explained variation is also computed and represents a measure equivalent to $R^{2}$ in linear regression. Standard errors were obtained by bootstrapping 200 resamples.

\section{Results \\ Prognostic performance}

Cox proportional hazard analyses using clinical and histological features with DMFS and BCSS as endpoints are summarized in Additional data file 1. Nine gene signatures were applied to the complete dataset of 1,127 breast cancer samples (Figure 2). Each of the signatures was used to define a poor outcome group and a good outcome group. For both clinical endpoints (DMFS, BCSS), the Kaplan-Meier curves obtained for each of the nine signatures were very similar - the exception being the Intrinsic/UNC gene set, which, per definition, defines five molecular subtypes (Figure 2a; see also Additional data file 2, Figure S1a). The log-rank test for differences in survival between the different groups as classified by the individual signatures was significant $(P<0.05)$ in all cases.

In addition, multivariate Cox proportional hazard models were fitted with lymph node (LN) status, size, estrogen receptor (ER) status (DMFS only) and each signature separately (see Additional data file 1, Table S2). All nine signatures remained as significant variables in the multivariate models for DMFS. Tumor size was the most significant, in terms of $P$ value, in all models except for those including the Intrinsic/UNC gene set signature and the 76-gene signature. When considering BCSS, three signatures remained significant: Intrinsic/UNC gene set $(P=0.01)$, Invasiveness Gene Signature $(P=0.02)$ and Core Serum Response signature $(P=0.039)$. The clinical variables LN and size were significant in all models considered.

The data were then divided into subsets according to LN status, ER status, grade and origin, and the signatures were compared in terms of their association with survival in these clinical subgroups (Figure 2b (top panel); see also Additional data file 2, Figure S1b (top panel)). With DMFS as the endpoint, no signature was significantly associated with survival in the ERnegative subgroup. In the LN-positive subgroup, some signatures were significantly associated with survival - while in the high-grade subgroup, only a single signature was significantly associated with DMFS. The sample sizes in these subgroups are relatively small ( $<200$ patients); however, the individual hazard ratios (HRs) for the signatures within these subgroups are of similar magnitude. For BCSS, only the ER-positive subgroup showed association and was the largest subgroup available for this endpoint $(n=297)$. Other clinical subgroups in this analysis have small sample sizes (between 80 and 160 
Figure 2

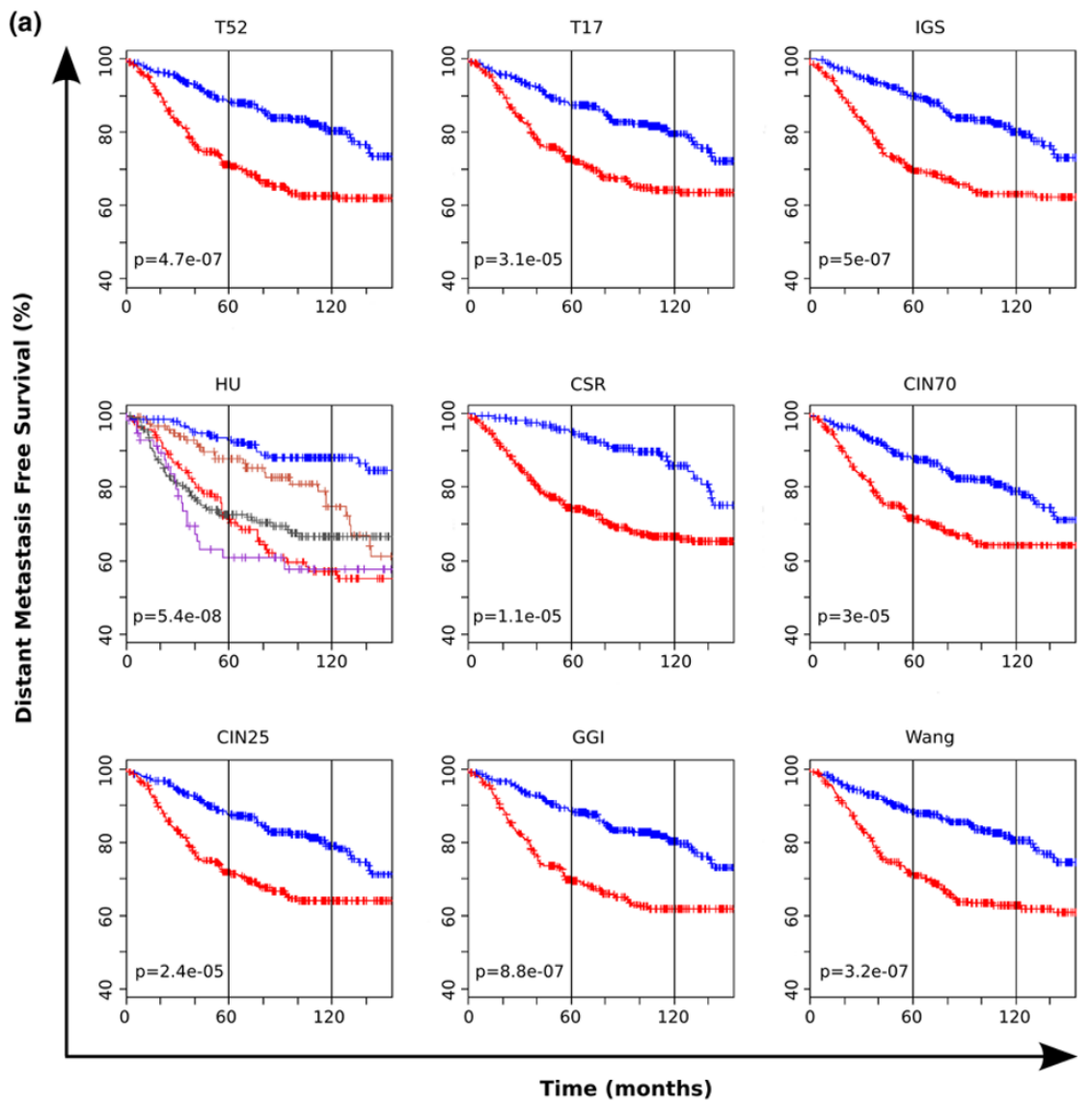

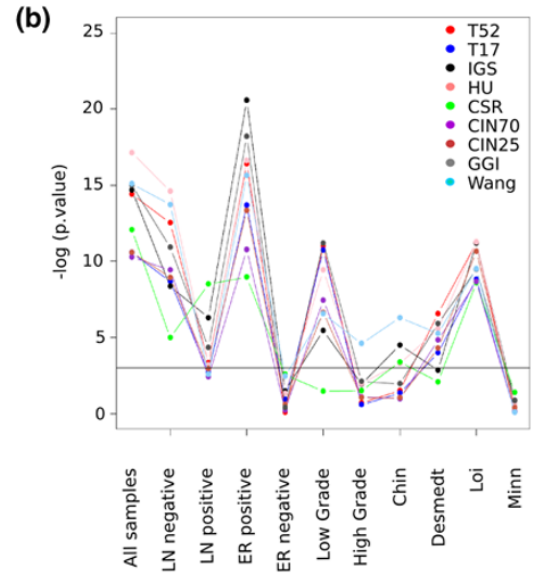

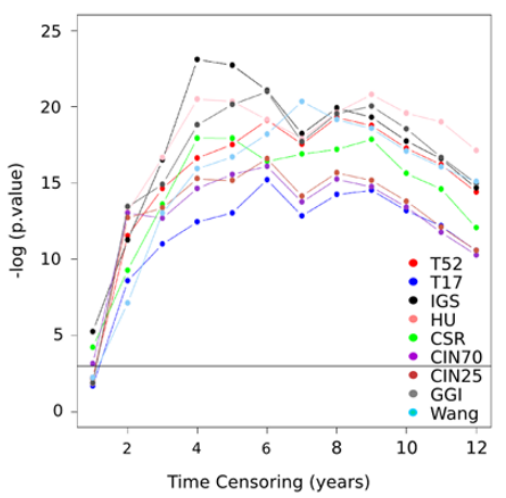

Kaplan-Meier analysis of distant metastasis-free survival. (a) Analysis performed for 724 patients. In all cases except the Intrinsic/UNC subtyping gene set $(\mathrm{HU})$ the blue (red) curve represents the good (poor) outcome group. Each panel depicts the result obtained after applying one of the classifiers (indicated in the heading) as described in Materials and methods. Represented signatures: T17 and T52, Molecular Prognostic Index signature [20]; IGS, Invasiveness Gene signature [16]; HU, Intrinsic/UNC gene set [15] - resulting in the Luminal A (blue), Normal-like (coral), Luminal B (red), Basal (grey) and Her2 (purple) subtypes - CSR, Core Serum Response signature [14]; CIN70 and CIN25, Chromosomal Instability signature [13]; GGl, Gene expression Grade Index [25]; and Wang, 76-gene signature [8]. (b) Top panel: performance of the signatures on subgroups of the patient population. Vertical axis, - $\log (P$ value $)$ of the log-rank test from the Kaplan-Meier analysis for a particular subgroup with distant metastasis-free survival (DMFS) as the endpoint. Analyzed subgroups: lymph node (LN)-negative, LN-positive, estrogen receptor (ER)-positive, ER-negative, low grade (Elston Ellis I), high grade (Elston Ellis III), individual Chin, Desmedt, Loi and Minn datasets. Horizontal line, $P=0.05$. Bottom panel: timecensoring performance analysis of the signatures. Horizontal axis, time at which right censoring was applied to all samples; vertical axis, -log $(P$ value $)$ of the log-rank test from the Kaplan-Meier analysis for a given time-censoring and a particular signature with DMFS as the endpoint. Horizontal line, $P=0.05$.

patients). We also applied the signatures to the individual datasets, and found that no signature showed association with survival in the Minn dataset (Figure 2b (top panel); see also Additional data file 2, Figure S1b (top panel)). For this dataset, only 80 samples had survival information; however, all signatures had HRs close to 1 .

The sensitivity of the signatures to variations in the time after which all events are censored was also investigated. Over a censoring range of 1 year to 12 years, each of the nine signatures showed a very similar pattern (Figure $2 \mathrm{~b}$ (bottom panel); see also Additional data file 2, Figure S1b (bottom panel)) specific to the clinical endpoint of interest. With DMFS as the endpoint, the performance measure reached a maximal value at approximately 5 years. This might be explained by the fact that some of the signatures were generated using a 5-year cutoff point to define the good and poor outcome groups. Two validation series of the 76-gene signature [9] and of the 70-gene signature [43] also showed strong time-dependent performance variability. The hazard ratio adjusted for clinical parameters, however, was still significant for the censoring time ranging from 1 year to 10 years.

\section{Classification concordance}

For each signature, every sample was labeled either good outcome or poor outcome. Figure 3 a provides an overview of the assignments of the samples to either outcome group by all signatures for DMFS (left panel) and for BCSS (right panel). 
(a)

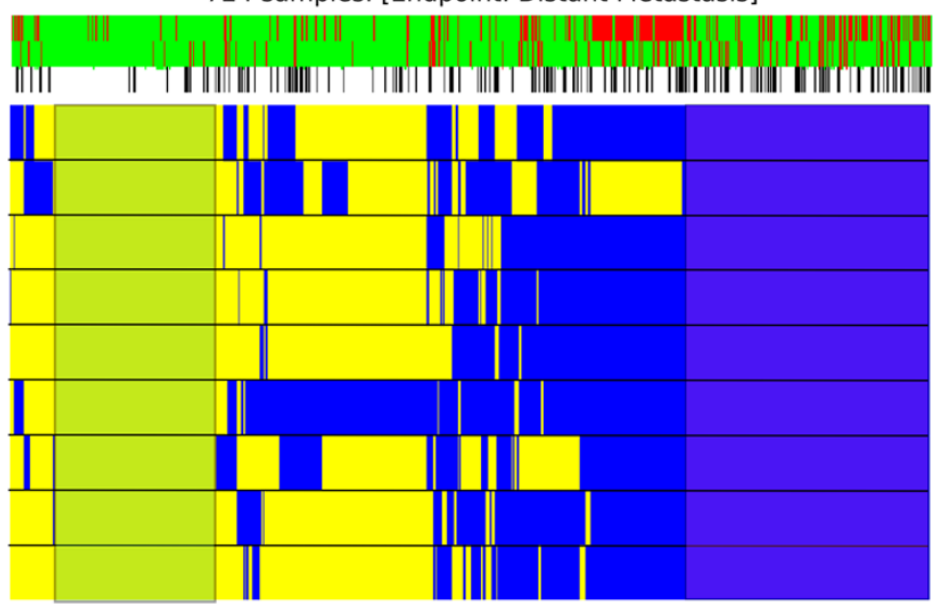

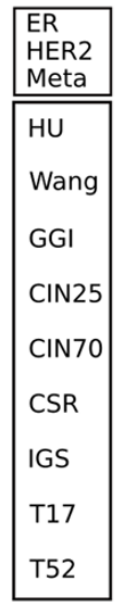

403 samples. [Endpoint: Death from Breast Cancer]

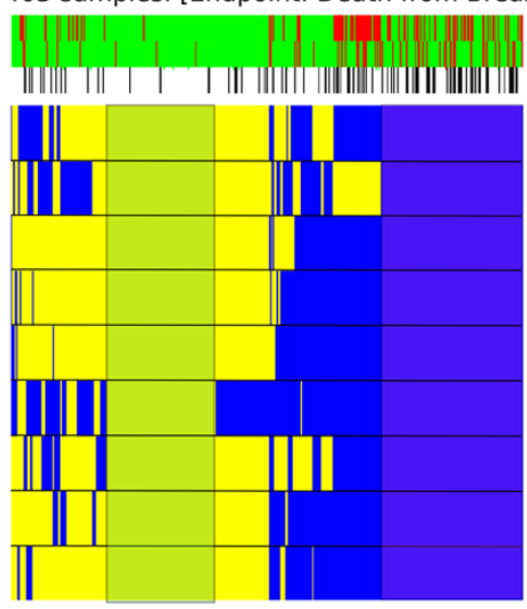

\begin{tabular}{|l|}
\hline ER \\
HER2 \\
Death \\
\hline \hline HU \\
Wang \\
GGI \\
CIN25 \\
CIN70 \\
CSR \\
IGS \\
T17 \\
T52 \\
\hline
\end{tabular}

(b)

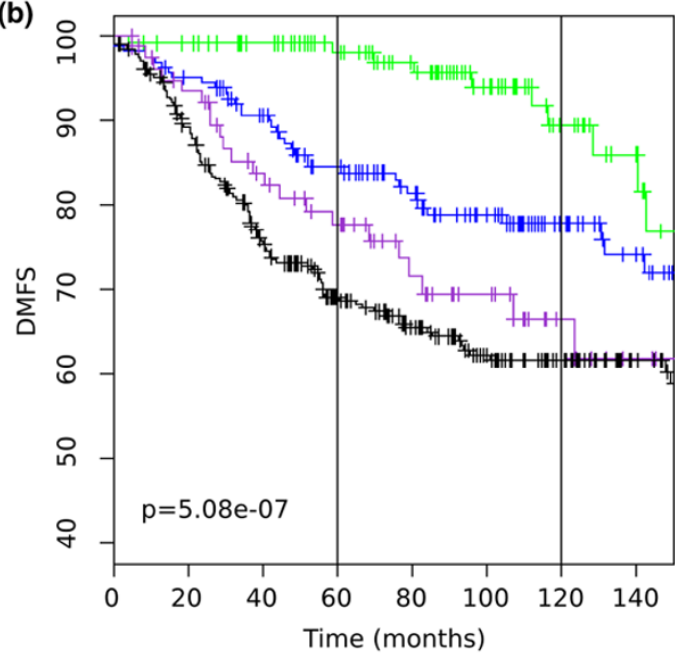

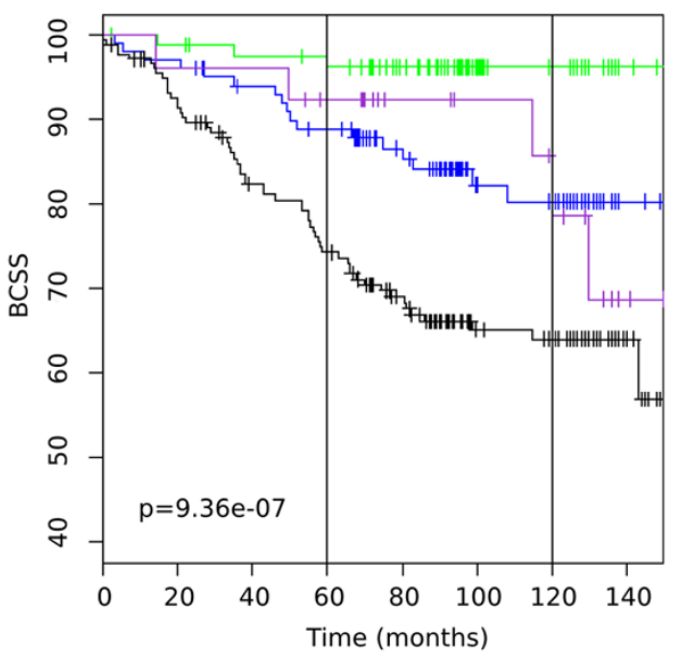

Classification and stratification of poor outcome for distant metastasis-free survival and breast cancer-specific survival. (a) Classification results of the nine gene signatures on the collection of 1,127 samples with distant metastasis-free survival (DMFS) (left panel) and breast cancerspecific survival (BCSS) (right panel) as the endpoints. In both panels, breast cancer samples are depicted in columns, and signatures in rows. Each cell colored according to the outcome of the signature in the corresponding row for the patient in the associated column: blue, poor outcome; yellow, good outcome. Top three lanes represent clinical parameters. HER2, HER2 status with red representing amplified cases and green nonamplified cases (as determined from gene expression); ER, estrogen receptor status with red representing ER-negative cases and green ER-positive cases as determined by gene expression; Meta or Death, DMFS or BCSS with events indicated in black. Represented signatures: HU, Intrinsic/UNC gene set [15]; IGS, Invasiveness Gene signature [16]; CIN70 and CIN25, Chromosomal Instability signatures [13]; GGI, Gene expression Grade Index [25]; T17 and T52, Molecular Prognostic Index signature [20]; CSR, Core Serum Response signature [14]; and Wang, the 76-gene signature [8]. For the subtyping based on the Intrinsic/UNC gene set, assignment to the Luminal B, Basal and HER2 subtypes was scored as poor outcome while assignment to the Luminal A and Normal types was scored as good outcome. Shaded regions highlight those patients classified as good outcome or poor outcome by all signatures. (b) Kaplan-Meier curves for the population of 1,127 samples stratified according to the number of times a sample is assigned to the poor outcome category with DMFS (left panel) and BCSS (right panel) as the endpoints. Graphs depict the curves for four subgroups: all good group (no poor outcome assignments; green), mainly good group (one to three poor outcome assignments; blue), mainly poor group (four to six poor outcome assignments; purple), and poor group (seven to nine poor outcome assignments; black).

We investigated the concordance of the classification labels for the nine signatures. Considering DMFS as the endpoint, only 322 out of 724 samples (44\%) had perfect concordance: 127 samples (17.5\%) were consistently classified as good outcome, while 195 samples (26.9\%) were classified as poor outcome by all signatures. These samples are indicated by the shaded regions in Figure 3a. A total of 114 samples (15.7\%) proved difficult to classify since more than one-third of the sig- natures assigned discordant labels to these samples. When BCSS was taken as the endpoint $(n=403)$, similar results were found: 198 (49\%) had perfect concordance, with 86 samples (21.3\%) always classified as good outcome and 112 samples (27.8\%) always classified as poor outcome by the signatures. A number of samples (46 samples, 11.4\%) were again difficult to classify, with more than one-third of the signatures assigning discordant labels to them. In order to investi- 
gate the effect of sample heterogeneity on the discordance, we repeated this analysis for subgroups based on ER status, LN status, and their combinations. The results are depicted in Additional data file 3 . This analysis revealed that the concordance/discordance percentages within these subgroups are always similar. That is, the discordance ranged from $52 \%$ to $62 \%$ for DMFS and from $44 \%$ to $60 \%$ for BCSS, leading to the conclusion that the concordance/discordance is not due to these clinical parameters.

Several clinical parameters are associated with the number of poor outcome assignments. The results for DMFS are depicted in Figure 4, while the results for BCSS are presented in Additional data file 4. In both cases, the proportion of events was significantly correlated with the number of times each sample was assigned to the poor outcome group (DMFS, chisquare test, $P=1.9 \times 10^{-4}$; BCSS, chi-square test, $P=6.1 \times$ 10-10). Similarly, the proportion of patients with ER-negative status (DMFS, $P=2.2 \times 10^{-16}$; BCSS, $P=2 \times 10^{-14}$ ), with HER2-positive status (DMFS, $P=8.5 \times 10^{-5}$; BCSS, $P=$ 0.0001 ), in the high-grade subgroup (DMFS, $P=2.2 \times 10^{-16}$; BCSS, $P=2.2 \times 10^{-16}$ ) and with a mean tumor size (DMFS, $P=0.0004$; BCSS, $P=2.4 \times 10^{-5}$ ) were all correlated to the number of times a sample was classified as poor outcome by the nine signatures.

To further investigate the impact of the number of poor outcome classifications, the samples were divided into four categories: all good (no poor outcome), mainly good (one to three poor outcomes), mainly poor (four to six poor outcomes), and poor (seven or more poor outcomes). Using this grouping, Kaplan-Meier analysis was performed using DMFS as the endpoint (Figure 3b (left panel), log-rank, $P=5.1 \times 10^{-7}$ ). A univariate Cox proportional hazard model showed significantly increased HRs when comparing the all good group with the other groups (versus mainly good group, $\mathrm{HR}=2.15$ (1.11 to 4.14); versus mainly poor group, $\mathrm{HR}=3.49$ (1.73 to 7.01 ); versus poor group, $\mathrm{HR}=4.12$ (2.27 to 7.49$)$ ). Using $B C S S$ as the endpoint, the log-rank test was again significant $(P=9.4$ $\times 10^{-7}$; Figure $3 \mathrm{~b}$ (right panel)) and the HRs increased significantly when comparing the all good group with the other groups (data not shown).

The sensitivity, specificity, positive predictive value, negative predictive value and predictive accuracy were very similar for all signatures - except for the Core Serum Response signature, which showed higher sensitivity and lower specificity (see Additional data file 1). The predictive accuracy (fraction of correctly assigned samples) of each signature was analyzed as a function of the number of times a sample was assigned to the poor outcome group. For both endpoints, the predictive accuracy is very high for the samples always classified as good outcome (DMFS, 87\%; BCSS, 96\%) and decreases dramatically with the number of poor outcome assignments. The predictive accuracy for the samples with nine poor out- come labels was only $45 \%$ for DMFS and $36 \%$ for BCSS (see Figure 4 and Additional data file 4).

\section{Enlarged gene signature analysis}

The intersection of seven gene signatures showed that no probe set was present in more than four signatures and that most of the probe sets were found in only one signature. In order to better reveal the common processes associated with the signatures, each of the signatures was enlarged by augmenting them with highly correlated probes (absolute Spearman correlation $>0.7$ ). These enlarged signatures contain, on average, three times as many probes as the original signatures. We identified a group of 72 probe sets present in at least five enlarged gene signatures. This overlap is highly significant, since observing an overlap of 72 or more probes between the two enlarged signatures with the smallest number of genes (164 genes, enlarged Invasiveness Gene Signature; and 189 genes, enlarged Gene expression Grade Index signature) is already highly significant $\left(P<2.2 \times 10^{-16}\right.$, hypergeometric test). The chance of observing an overlap of 72 or more probes among all seven enlarged signatures will be even smaller, and thus even more significant. An enrichment analysis of these 72 probe sets showed an overrepresentation of genes related to DNA replication, cell cycle, and proliferation.

\section{Constructing and validating module classifiers}

Functionally related gene sets were merged to define functional modules. This resulted in the following 11 modules: Immune, KRAS, Proliferation1 (defined by genes common to two to four enlarged signatures), Proliferation2 (defined by genes common to five or more enlarged signatures), RNA splicing, Rb pathway, Sterol biosynthesis, Extracellular matrix constituent (ECM), Focal adhesion, Negative regulation of proliferation, and Apoptosis (Figure 5a). For each of these modules we determined a nearest centroid classifier on the Chin-Loi training set. On this training set, with DMFS as the endpoint, all modules except Sterol biosynthesis had a significant association with outcome (log-rank test $P$ value ranging from $4.4 \times 10^{-3}$ to $6.8 \times 10^{-9}$; Figure $5 b$ ).

We then set out to construct a classifier that combines the binary class assignments of the module classifiers into a single, three-valued (low-risk, intermediate-risk and high-risk) classification output. We restricted the search to pairs of modules, since the size of the discordant groups (where at least one module classifier output differs from the others) grows as the number of modules combined increases, leading to difficulty in interpretability. The separate classifiers were combined in a pairwise fashion (55 possible combinations) and the resulting classifiers were evaluated on the Chin-Loi training set. The classifier with the most significant log-rank test (lowest $P$ value) on the training data was selected. This classifier was based on the Immune and RNA splicing modules (see Additional data files 5 and 6 ). Samples that are assigned to the 
Figure 4

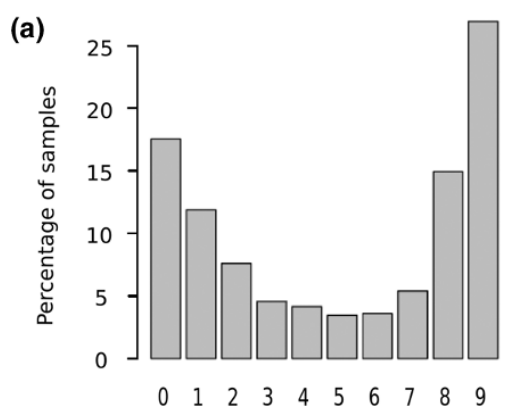

Number of "poor" assignments

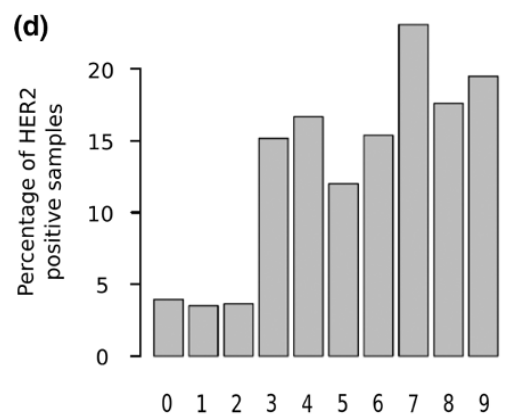

Number of "poor" assignments

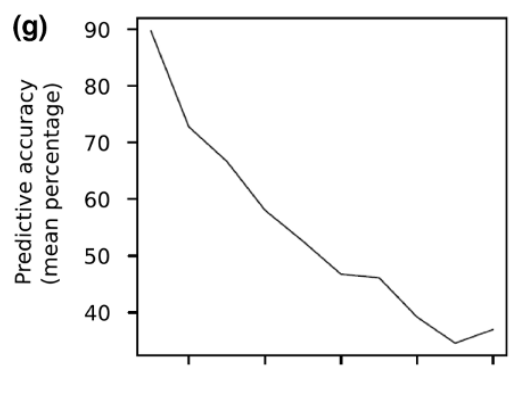

$\begin{array}{llllllllll}0 & 1 & 2 & 3 & 4 & 5 & 6 & 7 & 8 & 9\end{array}$

Number of "poor" assignments

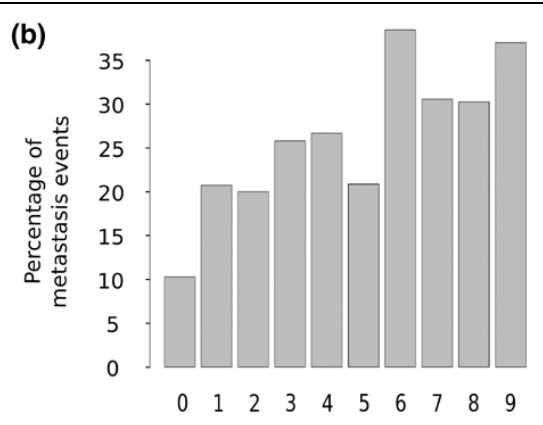

Number of "poor" assignments

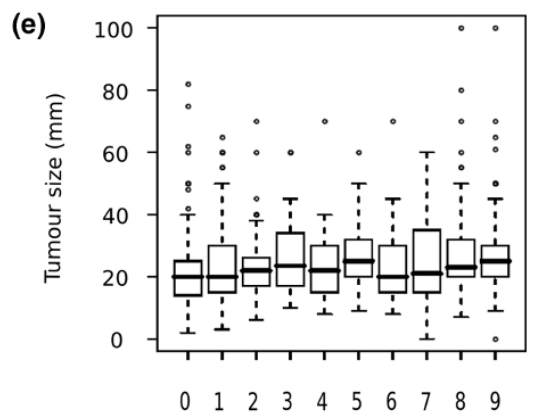

Number of "poor" assignments

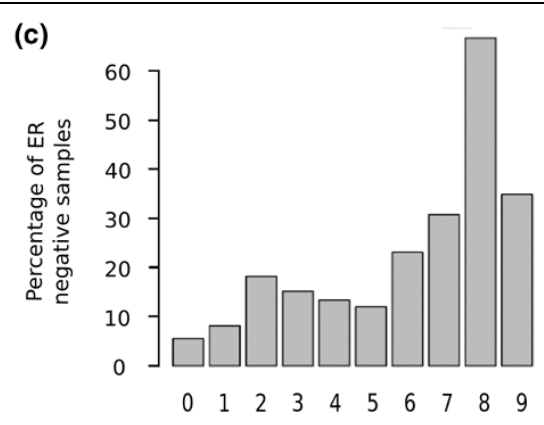

Number of "poor" assignments

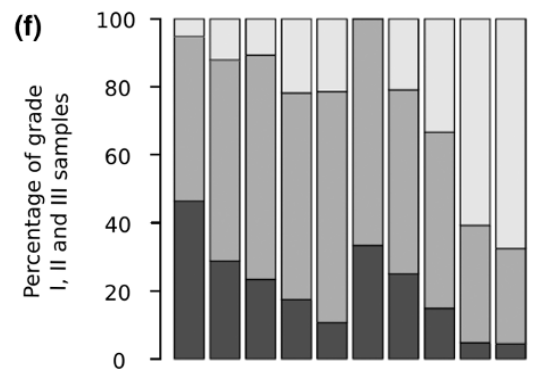

$\begin{array}{llllllllll}0 & 1 & 2 & 3 & 4 & 5 & 6 & 7 & 8 & 9\end{array}$

Number of "poor" assignments

Overlap and performance analysis of $\mathbf{7 2 4}$ samples with distant metastasis-free survival as the endpoint. (a) Distribution of the samples as a function of the number of times a sample was classified as of poor prognosis by the gene signatures. (b) Distribution of metastasis events as a function of the number of times a sample was classified as of poor prognosis by the gene expression signatures. (c) Distribution of estrogen receptor (ER)-negative samples as a function of the number of times a sample was classified as of poor prognosis by the gene signatures. (d) Distribution of HER2 amplified samples as a function of the number of times a sample was classified as of poor prognosis by the gene signatures. (e) Tumor size (mean, 5th, 25th, 75th and 95th percentiles) as a function of the number of times a sample was classified as of poor prognosis by the gene signatures. (f) Distribution of grade I, grade II and grade III samples (Elston Ellis) as a function of the number of times a sample was classified as of poor prognosis by the gene signatures. Dark shading, grade I; grey shading, grade II; pale shading, grade III. (g) Average predictive accuracy (percentage of samples well classified) as a function of the number of times a sample was classified as of poor prognosis by the gene signatures.

good outcome (poor outcome) category by both the Immune and the RNA splicing module classifiers are classified as low (high) risk, while the discordant cases are assigned to the intermediate risk category. On independent data, this classifier was a significant prognosis predictor (data not shown) of DMFS (dataset of Desmedt and colleagues [9]) and BCSS (datasets of Pawitan and colleagues [28] and Miller and colleagues [18]).
The Immune and RNA splicing classifier was then validated using the dataset of van de Vijver and colleagues [7]. For both DMFS and BCSS, we found a highly significant association between the classifier and outcome (log-rank test: DMFS, $P=$ $2.28 \times 10^{-8}$; BCSS, $P=1.8 \times 10^{-10}$ ) (Figure 5c). For DMFS, the HR for the intermediate-risk group versus the low-risk group is 2.25 (1.3 to 3.9 ), while the HR for the high-risk group versus the low-risk group is 4.35 (2.57 to 7.36 ). Similarly, for BCSS the HR of the intermediate-risk group versus the lowrisk group was 2.31 (1.2 to 4.5 ) and the HR for the high-risk- 


\section{Figure 5}

(a)

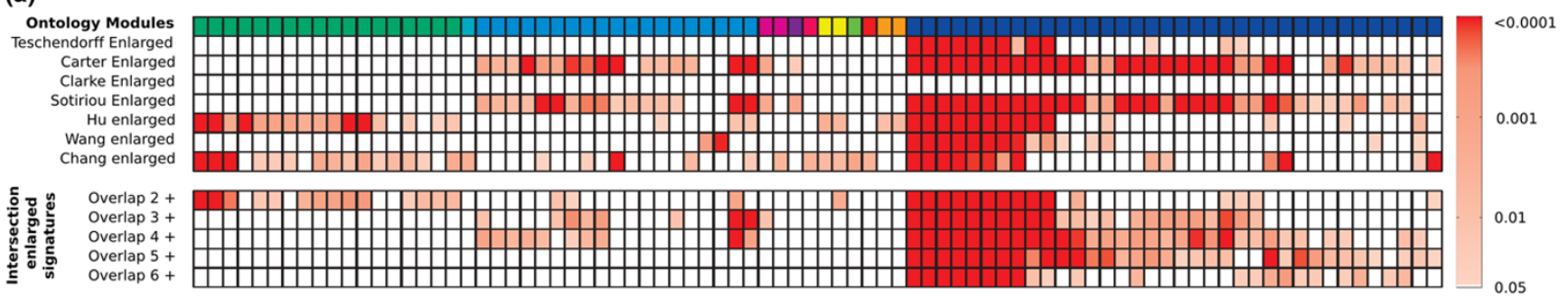

(b)
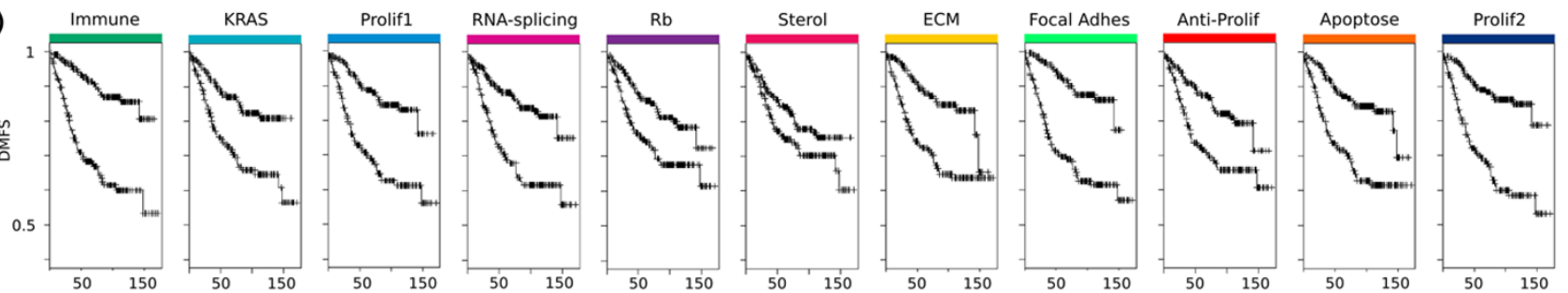

(c) RNA-splicing Immune classifier

RNA-splicing Immune classifier
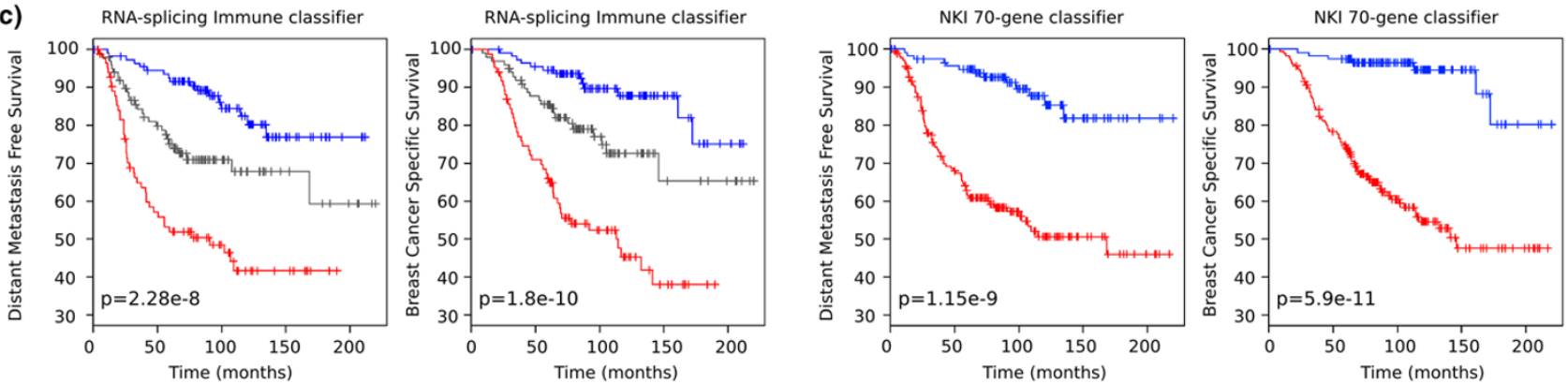

Enrichment analysis for the seven enlarged signatures and Kaplan-Meier analysis of distant metastasis-free survival. (a) Enrichment analysis for the seven enlarged signatures on a collection of 1,889 gene sets from the Reactome, Kyoto Encyclopedia of Genes and Genomes (KEGG), Molecular Signatures Database, and Gene Ontology databases. Significance of the $P$ values was computed with the hypergeometric test. All $P$ values were adjusted for multiple testing using the Benjamini-Hochberg method. Each cell in the matrix represents the adjusted $P$ value for a given enlarged signature and a gene set. The ontology modules to which a particular gene set belongs are indicated at the top. See (b) for the link between the color coding and the module identity. (b) Kaplan-Meier analysis of distant metastasis-free survival (DMFS) on the Chin-Loi training set for each of the 11 ontology modules. The Kaplan-Meier analysis is based on the output of a nearest mean classifier trained on the genes in each of the ontology modules. (c) Kaplan-Meier analysis of DMFS on van Vijver and colleagues' breast cancer series as stratified by the Immune and RNA splicing module classifier, for both endpoints. Blue curve, low-risk group where both classifiers assign a patient to the good outcome group; grey curve, intermediate-risk category where the classifiers are discordant; red curve, high-risk category where both classifiers assign a patient to the poor outcome group. For comparison, the same dataset as stratified by the Netherlands Cancer Institute (NKI) 70-gene classifier for both endpoints is also presented.

group versus the low-risk group is 6.1 (3.3 to 11.4$)$. The known clinical predictors Elston Ellis grading, age at diagnosis, size $(\mathrm{mm})$ and ER status, as well as the Immune and RNA splicing classifier, were then included in a multivariate analysis. For both endpoints, the combined classifier was the most significant variable in the model (see Additional data file 1).

The gain in predictive accuracy from adding the RNA splicing/ Immune signature to each of the three common clinical staging systems is presented in Table 2. The largest decrease in predictive inaccuracy is seen when the RNA splicing/lmmune signature is added to the St Gallen index, as this has the worst individual performance for both DMFS and BCSS ( 0.31 and 0.287 , respectively). In this case, the predictive accuracy is reduced from 0.287 to 0.254 for BCSS (DMFS, from 0.310 to
0.282). In terms of explained variation, the St Gallen index is again the worst, explaining only $1 \%$ to $2 \%$ of the variation in this dataset. With $6 \%$ to $8 \%$ of the explained variation being attributable to the Nottingham Prognostic Index dataset, it is the best of the clinical staging systems. In contrast, the RNA splicing/Immune signature explains a larger amount of the variation $(10 \%$ to $13 \%)$ on its own, and including it with one of the three staging systems improves on this only marginally. For this dataset, there is a gain in the predictive accuracy when adding the RNA splicing/Immune classifier to existing predictors. Additional data file 7 shows the prognostic capacity of the signature conditional on the levels of the different clinical staging systems. 
Table 2

Explained variation and predictive inaccuracy for distant metastasis-free survival and breast cancer-specific survival in the 295 Netherlands Cancer Institute dataset

\begin{tabular}{|c|c|c|c|c|}
\hline \multirow[t]{2}{*}{ Model } & \multicolumn{2}{|c|}{ Distant metastasis-free survival } & \multicolumn{2}{|c|}{ Breast cancer-specific survival } \\
\hline & Predictive inaccuracy & Explained variation (\%) & Predictive inaccuracy & Explained variation (\%) \\
\hline No predictors & $0.314 \pm 0.02$ & & & $0.292 \pm 0.02$ \\
\hline Adjuvant online & $0.303 \pm 0.02$ & $3.5 \pm 1.8$ & $0.274 \pm 0.02$ & $6.1 \pm 2.0$ \\
\hline Nottingham Prognostic Index & $0.294 \pm 0.02$ & $6.2 \pm 2.4$ & $0.270 \pm 0.02$ & $7.8 \pm 2.6$ \\
\hline St Gallen & $0.310 \pm 0.02$ & $1.2 \pm 0.9$ & $0.287 \pm 0.02$ & $1.9 \pm 1.0$ \\
\hline RNA splicing/Immune module classifier & $0.282 \pm 0.02$ & $9.9 \pm 3.0$ & $0.254 \pm 0.02$ & $13.0 \pm 3.8$ \\
\hline Adjuvant online and module classifier & $0.281 \pm 0.02$ & $10.3 \pm 2.9$ & $0.250 \pm 0.02$ & $14.5 \pm 3.8$ \\
\hline $\begin{array}{l}\text { Nottingham Prognostic Index and module } \\
\text { classifier }\end{array}$ & $0.277 \pm 0.02$ & $11.6 \pm 3.2$ & $0.248 \pm 0.02$ & $15.2 \pm 3.8$ \\
\hline St Gallen and module classifier & $0.282 \pm 0.02$ & $9.9 \pm 3.0$ & $0.254 \pm 0.02$ & $13.1 \pm 3.7$ \\
\hline $\begin{array}{l}\text { Gain by adding module classifier to Adjuvant } \\
\text { online }\end{array}$ & 0.022 & 6.8 & 0.024 & 8.4 \\
\hline $\begin{array}{l}\text { Gain by adding module classifier to } \\
\text { Nottingham Prognostic Index }\end{array}$ & 0.017 & 5.4 & 0.022 & 7.4 \\
\hline Gain by adding module classifier to St Gallen & 0.028 & 8.7 & 0.033 & 11.2 \\
\hline
\end{tabular}

Data presented as the mean \pm standard error.

\section{Discussion}

The analyses performed here demonstrate that each of the nine gene expression signatures have similar classification performance based on the sensitivity, specificity, negative predictive value, positive predictive value and predictive accuracy. All gene expression signatures added independent information to a multivariate model including standard pathological and clinical criteria. Although the gene expression classifiers were mostly defined to determine the risk of distant metastasis events and not the risk of death from breast cancer, we found similar results for each gene classifier when using either DMFS or BCCS as the endpoint.

The Gene expression Grade Index [25] and the molecular prognostic index signatures (T17 and T52) [20] were developed for ER-positive breast cancer, while the 76-gene expression classifier signature [8] was defined for LN-negative tumors. Despite these prerequisites, we applied the nine gene expression classifiers to the same dataset without any consideration for the heterogeneity of these samples as our first goal was to compare these different classifiers when applied to the same dataset. More importantly, the generalization of subgroup-specific classifiers (for example, LNO classifiers) across a complete cohort of breast cancer samples (both LNO and LN1) hints at the existence of common biological processes determining the outcome. We were interested in revealing these processes. Furthermore, when evaluating the signatures in specific subgroups of patients, we showed similar behavior for each of these nine gene signatures. No signature showed strong association with survival when applied to LN-positive,
ER-negative or high-grade subgroups. These results are potentially explained by the fact that these factors identify a set of intrinsically poor outcome cases; that is, they contain no good outcome cases. This emphasizes the fact that gene expression classifiers should, in our opinion, not be regarded as a tool to replace standard pathological and clinical criteria, but should instead be integrated with clinical parameters. Gene expression classifiers can be employed to improve stratification in subgroups of breast cancer patients with good prognosis, where the groups are defined based on standard pathological and clinical criteria.

Fan and colleagues [24] showed similar performance and significant concordance between the 70 -gene signature $[6,7]$, the Core Serum Response signature [14], the Genomic Health signature [10] and the Intrinsic/UNC gene set [15] when applied to the dataset of van de Vijver and colleagues [7]. In contrast, we show here that agreement between gene expression signatures is low, with $>50 \%$ of the samples having at least one discordant class assignment. We showed that the predictive accuracy dramatically decreases with the number of poor prognostic assignments a sample receives. The best classification performance was obtained for the group of patients with only good outcome assignments. These results immediately reveal the dilemma faced by a patient diagnosed with breast cancer, and determines consultation of a collection of signatures to predict disease outcome. The result obtained is uncertain in almost $50 \%$ of the cases. As our results were less optimistic than those of Fan and colleagues [24], we repeated our analyses as described above but this 
time used the dataset of van de Vijver and colleagues [7] and the following signatures: the 70-gene signature [6,7] (employing Fan and colleagues' labeling [24]), the Core Serum Response signature [14], the Genomic Health signature [10], the Intrinsic/UNC gene set [15], and the Gene expression Grade Index [25]. The results recapitulated our earlier results. In particular, only $42 \%$ of the samples received a concordant class assignment, while the ER status, HER2 status, pathological grading and tumor size were all correlated with the number of times a sample was classified as poor outcome by the signatures. As was demonstrated earlier, the predictive accuracy decreased with the number of poor outcome assignments. Larger datasets (such as those acquired in the TAILORx and MINDACT trials $[11,12]$ are required to shed more light on the cases where the signatures give discordant class assignments.

To gain more insight into the small degree of overlap between the genes comprising the different classifiers, we generated an enlarged signature for each signature. The intersection of the enlarged signatures identified a core of 72 genes significantly enriched in DNA replication, cell cycle and mitosis ontology annotations, which we consider the common background of these gene signatures. Proliferation genes are a major component of many prognostic signatures in breast carcinoma and other tumor types [44]. Among the 72 genes we found AURKA, BIRC5, CCNB1, MKI67 and MYBL2, which define the complete set of proliferation genes from the Genomic Health signature [10]. The proliferation modules also contain genes frequently described as markers of proliferation in different types of cancer [45]: PLK1, BUB1, CCNA2, CCNB1, CCNB2, CCNE2, FOXM1, and TOP2A. These genes are derived from the functional intersection of the enlarged gene expression signatures, indicating that proliferation is a major driver of the prognosis gene signatures.

The enrichment analysis of the enlarged signatures revealed 11 gene ontology modules. Identification of distinct biological processes correlated with survival or other clinicopathological features is a major step towards improving our understanding of tumor development and to providing accurate information to develop new targeted therapies. Yu and colleagues generated 500 gene signatures of ER-positive and ER-negative tumors [46], and found the following pathways to be overrepresented in the signatures: apoptosis, proliferation, focal adhesion, RNA splicing and immunity. They emphasized that similar pathways are common to different gene signatures, whereas the individual genes defining these pathways can still have varying degrees of association with outcome.

We showed that the combination of the Immune and RNA splicing modules define a classifier that is highly accurate in predicting both DMFS and BCSS. In addition, the classifier showed an improvement in predictive accuracy when combined with commonly used clinical staging systems. This indi- cates that not only proliferation but also other functional processes have prognostic power. Teschendorff and colleagues recently showed that the overexpression of a sevengene immunity module is associated with good outcome in 186 ER-negative breast cancers [21]. No significant correlation between lymphocyte infiltration and this immunity module was found. Two of these seven genes (XCL2 and HLA-F) are also in our classifier. Recent clinical and experimental studies have revealed that not only cancer cell intrinsic processes, but also cancer cell extrinsic processes - including angiogenesis, remodeling of the extracellular matrix, and inflammation - are critical in determining malignant outcome. The role of the immune system during cancer progression has recently gained much attention [47]. The reciprocal interaction between the immune system and cancer can be regarded as a double-edged sword: whereas certain interactions inhibit or prevent cancer growth, other interactions actually contribute to tumor progression. For example, in situ analysis of tumorinfiltrating lymphocytes in human colorectal cancer samples revealed that the influx of $\mathrm{T}$ lymphocytes is associated with improved survival, and the immunological data were found to be a better predictor of patient survival than the histopathological methods currently used to stage colorectal cancer [48].

The RNA splicing process is a key molecular event in the generation of protein biodiversity. Alteration of the normal process results in the production of altered mRNA or in an off-balance production of tissue-specific mRNA isoforms [49-51]. The main consequences of this abnormal RNA splicing process are a reduction of the normal protein level or the production of abnormal proteins. Aberrant mRNA splicing variants are found in many cancers and can interfere with major biological events such as apoptosis, cell-cycle control, adhesion, differentiation or angiogenesis. Mutations in splicing cis-acting sequences have been associated with the BRCA1 gene in breast cancer [52] and the KIT oncogene in gastrointestinal stromal tumor [53]. The RNA splicing module we identified contains several genes that are individually strongly associated with survival. More specifically, SFRS10 is significantly overexpressed in breast cancer and might be responsible for splicing of CD44 isoforms associated with tumor progression and metastasis [54]. SRPK1 is upregulated in breast cancer and its expression level is proportional to the tumor grade. Inhibition of $S R P K 1$ results in reduced phosphorylation of MAPK3, MAPK1 and AKT [55]. Targeted SRPK1 treatment seems to be a promising way to increase apoptosis, to decrease proliferation and to enhance the sensitivity to chemotherapeutics drugs [56]. LSM1 is located at 8p11-12 loci and is amplified in almost $20 \%$ of breast cancer cases [57]. Streicher and colleagues showed that overexpression of $h L s m 1$ transforms mammary epithelial cells, and inhibition of its expression in breast cancer cells reduces anchorage-independent proliferation [58]. Yang and colleagues similarly showed the same ability of LSM1 to transform human mammary epithelial cells in vitro [57]. 


\section{Conclusion}

In the present article we set out to address three questions regarding the signatures considered in the study. First, we can conclude that the nine gene expression signatures had similar performance. This was observed for both the accuracy with which samples were assigned to the dichotomous poor/good outcome groups as well as the level of association with survival found. Nevertheless, the concordance of outcome assignment between gene expression signatures is low, with $50 \%$ of the samples receiving at least one outcome assignment that is discordant with the assignments of the other signatures. This relatively high level of gene expression classification instability is associated with a dramatic decrease in predictive accuracy with an increase in the number of poor outcome assignments. The heterogeneity in the outcome assignments of the different classifiers can most probably be attributed to the different approaches that were followed to construct the classifiers, the heterogeneity in the sample populations employed to construct the classifiers, and sample size issues [59].

In the present study we showed that the common background of the nine gene signatures investigated is a 72-gene cluster. Eleven gene ontology modules were overrepresented in the enlarged signatures. These modules revealed a wide array of functional groups that were overrepresented in gene sets highly correlated with the probes contained in the original signatures. Finally, we demonstrated that the combination of the Immune and RNA splicing modules defined an efficient classifier for breast cancer. This result shows that pathway-level analysis of microarrays is able to provide a functionally coherent and highly efficient prognosis classifier, which will most probably be more stable than the classifiers from which it originates.

\section{Competing interests}

LvV is employed by Agendia BV, has an ownership interest in MammaPrint, and is the named inventor on a patent to use microarray technology to ascertain breast cancer prognosis and holds equity interests in Agendia BV. The other authors declare that they have no competing interests.

\section{Authors' contributions}

FR and MHvV contributed equally to this work, carrying out the study design, data mining, data analysis and manuscript writing. NJA carried out the study design, data mining, data analysis and manuscript writing. $\mathrm{HMH}$ carried out the study design and data mining. KEdV carried out the data analysis and manuscript writing. MK carried out the study design and manuscript writing. AET carried out the Molecular Prognosis Index calculation. SM carried out the study design and manuscript advising. LvV carried out the manuscript advising. CC carried out the Molecular Prognosis Index calculation. RJS carried out the fund raising and manuscript advising. MJvdV carried out the study design, data analysis and manuscript writing. LFAW carried out the study design, data mining, data analysis and manuscript writing.

\section{Additional files}

The following Additional files are available online:

\section{Additional file 1}

A Word file containing the supplementary Materials and methods section and four supplementary tables. Table $\mathrm{S} 1$ lists data for multivariate and univariate Cox regression analyses with DMFS and BCSS as the endpoints. Table S2 lists data for multivariate Cox regression analysis with selected clinical parameters ER status based on immunohistochemistry (DMFS only), LN status (positive versus negative), histological grading (Elston Ellis I, II and III) - and the output of the nine gene expression classifiers as input and either DMFS and BCSS as clinical endpoints. Table S3 lists a performance analysis of the signatures on the complete set of 1,127 patients with dichotomous outcome labels of poor outcome and good outcome derived from DMFS and BCSS. Table S4 lists data for multivariate Cox regression analysis with selected clinical parameters ER status based on immunohistochemistry, LN status (positive versus negative), histological grading (Elston Ellis I, II and III) - tumor size and the output of the Immune and RNA splicing modules gene signature (IR) or the 70gene signature (NKI) as the input and DMFS and BCSS analysis as the clinical endpoint.

See http://www.biomedcentral.com/content/ supplementary/bcr2192-S1.doc

\section{Additional file 2}

An Adobe file containing a figure of the RNA splicing, Immune and 72 Proliferation gene annotations [Probe_ID, EntrezID, OMIM, Ensembl, UnigeneID, Representative Public ID, RefSeq Transcript ID, Gene Symbol, k-means metastasis, k-means no metastasis]. See http://www.biomedcentral.com/content/ supplementary/bcr2192-S2.png

\section{Additional file 3}

An Adobe file containing a figure showing the nine signature Kaplan-Meier curves with BCSS as endpoints (S1a), showing the performance of the signatures on subgroups of the patient population (S1b (top panel)), and showing) the time-censoring performance analysis of the signatures ( $1 \mathrm{~b}$ (bottom panel).

See http://www.biomedcentral.com/content/ supplementary/bcr2192-S3.pdf 


\section{Additional file 4}

An image file containing a figure showing heatmaps of the concordance of the nine classifiers across clinical subgroups among the 1,127 human breast tumor samples.

See http://www.biomedcentral.com/content/ supplementary/bcr2192-S4.png

\section{Additional file 5}

An image file containing a figure showing the overlap and performance analysis of 403 samples with BCSS as the endpoint.

See http://www.biomedcentral.com/content/

supplementary/bcr2192-S5.png

\section{Additional file 6}

An Excel file containing a figure showing the log-rank test $P$ value on the Chin-Loi training set for each pairwise combination of the module classifiers.

See http://www.biomedcentral.com/content/

supplementary/bcr2192-S6.xls

\section{Additional file 7}

An Adobe file containing a figure showing the KaplanMeier plots on the dataset of van de Vijver and colleagues for the subgroups defined by the Nottingham Prognostic Index, St Gallen, and AdjuvantOnline! clinical staging systems - plots for the Immune/RNA splicing module classifier within each of the clinical subgroups for each staging system.

See http://www.biomedcentral.com/content/ supplementary/bcr2192-S7.pdf

\section{Acknowledgements}

FR was supported by grants from the French 'Association pour la Recherche contre le Cancer' and the French Academy of Medecine. The present article is dedicated to $\mathrm{JH}$ Petersen.

\section{References}

1. Goldhirsch A, Wood WC, Gelber RD, Coates AS, Thürlimann B, Senn $\mathrm{H}$-J: Progress and promise: highlights of the international expert consensus on the primary therapy of early breast cancer 2007. Ann Oncol 2007, 18:1133-1144.

2. Eifel P, Axelson JA, Costa J, Crowley J, Curran WJ, Deshler A, Fulton S, Hendricks CB, Kemeny M, Kornblith AB, Louis TA, Markman $M$, Mayer R, Roter D: National Institutes of Health Consensus Development Conference Statement: adjuvant therapy for breast cancer, November 1-3, 2000. J Natl Cancer Inst 2001, 93:979-989.

3. Blamey RW, Ellis IO, Pinder SE, Lee AHS, Macmillan RD, Morgan DAL, Robertson JFR, Mitchell MJ, Ball GR, Haybittle JL, Elston $\mathrm{CW}$ : Survival of invasive breast cancer according to the Nottingham Prognostic Index in cases diagnosed in 1990-1999. Eur J Cancer 2007, 43:1548-1555.

4. Ravdin PM, Siminoff LA, Davis GJ, Mercer MB, Hewlett J, Gerson $\mathrm{N}$, Parker HL: Computer program to assist in making decisions about adjuvant therapy for women with early breast cancer. $J$ Clin Oncol 2001, 19:980-991.
5. Early Breast Cancer Trialists' Collaborative Group: Effects of chemotherapy and hormonal therapy for early breast cancer on recurrence and 15-year survival: an overview of the randomised trials. Lancet 2005, 365:1687-1717.

6. van 't Veer LJ, Dai H, Vijver MJ van de, He YD, Hart AAM, Mao M, Peterse HL, Kooy K van der, Marton MJ, Witteveen AT, Schreiber GJ, Kerhoven RM, Roberts C, Linsley PS, Bernards R, Friend SH: Gene expression profiling predicts clinical outcome of breast cancer. Nature 2002, 415:530-536.

7. Vijver MJ van de, He YD, van 't Veer LJ, Dai H, Hart AAM, Voskuil DW, Schreiber GJ, Peterse JL, Roberts C, Marton MJ, Parrish M, Atsma D, Witteveen A, Glas A, Delahaye L, Velde T van der, Bartelink $\mathrm{H}$, Rodenhuis S, Rutgers ET, Friend $\mathrm{SH}$, Bernards R: A gene-expression signature as a predictor of survival in breast cancer. N Engl J Med 2002, 347:1999-2009.

8. Wang $Y$, Klijn JG, Zhang $Y$, Sieuwerts AM, Look MP, Yang F, Talantov D, Timmermans M, Meijer-van Gelder ME, Yu J, Jatkoe T, Berns EM, Atkins D, Foekens JA: Gene-expression profiles to predict distant metastasis of lymph-node-negative primary breast cancer. The Lancet 2005, 365:671-679.

9. Desmedt C, Piette F, Loi S, Wang Y, Lallemand F, Haibe-Kains B, Viale G, Delorenzi M, Zhang Y, d'Assignies MS, Bergh J, Lidereau R, Ellis P, Harris AL, Klijn JGM, Foekens JA, Cardoso F, Piccart MJ, Buyse M, Sotiriou C: Strong time dependence of the 76-gene prognostic signature for node-negative breast cancer patients in the TRANSBIG multicenter independent validation series. Clin Cancer Res 2007, 13:3207-3214.

10. Paik S, Shak S, Tang G, Kim C, Baker J, Cronin M, Baehner FL, Walker MG, Watson D, Park T, Hiller W, Fisher ER, Wickerham $\mathrm{DL}$, Bryant J, Wolmark N: A multigene assay to predict recurrence of tamoxifen-treated, node-negative breast cancer. $N$ Engl J Med 2004, 351:2817-2826.

11. Bogaerts J, Cardoso F, Buyse M, Braga S, Loi S, Harrison JA, Bines J, Mook S, Decker N, Ravdin P, Therasse P, Rutgers E, van 't Veer LJ, Piccart M, the TRANSBIG consortium: Gene signature evaluation as a prognostic tool: challenges in the design of the MINDACT trial. Nat Clin Pract Oncol 2006, 3:540-551.

12. Paik S: Development and clinical utility of a 21-gene recurrence score prognostic assay in patients with early breast cancer treated with tamoxifen. Oncologist 2007, 12:631-635.

13. Carter SL, Eklund AC, Kohane IS, Harris LN, Szallasi Z: A signature of chromosomal instability inferred from gene expression profiles predicts clinical outcome in multiple human cancers. Nat Genet 2006, 38:1043-1048.

14. Chang HY, Nuyten DS, Sneddon JB, Hastie T, Tibshirani R, Sorlie T, Dai H, He YD, van 't Veer LJ, Bartelink H, van Rijn M, Brown PO, Vijver MJ van de: Robustness, scalability, and integration of a wound-response gene expression signature in predicting breast cancer survival. Proc Natl Acad Sci USA 2005, 102:3738-3743.

15. Hu Z, Cheng F, Oh DS, Marron JS, He X, Qaqish BF, Livasy C, Carey LA, Reynolds E, Dressler L, Nobel A, Parker J, Ewend MG, Sawyer LR, Wu J, Liu Y, Nanda R, Tretiakova M, Orrico AR, Dreher D, Palazzo JP, Perreard L, Nelson E, Mone M, Hansen H, Mullins M, Quackenbush JF, Ellis MJ, Olopade OI, Bernard PS, Perou CM: The molecular portraits of breast tumors are conserved across microarray platforms. BMC Genomics 2006, 7:96.

16. Liu R, Wang X, Grace YC, Dalerba P, Gurney A, Hoey T, Sherlock $G$, Lewicki J, Shedden K, Clarke MF: The prognostic role of a gene signature from tumorigenic breast-cancer cells. $N$ Engl J Med 2007, 356:217-226.

17. Loi S, Haibe-Kains B, Desmedt C, Lallemand F, Tutt AM, Gillet C, Ellis $P$, Harris A, Bergh J, Foekens JA, Klijn JGM, Larsimont $D$, Buyse M, Bontempi G, Delorenzi M, Piccart MJ, Sotiriou C: Definition of clinically distinct molecular subtypes in estrogen receptor-positive breast carcinomas through genomic grade. $J$ Clin Oncol 2007, 25:1239-1246.

18. Miller LD, Smeds J, George J, Vega VB, Vergara L, Ploner A, Pawitan Y, Hall P, Klaar S, Liu ET, Bergh J: An expression signature for p53 status in human breast cancer predicts mutation status, transcriptional effects, and patient survival. Proc Natl Acad Sci USA 2005, 102:13550-13555.

19. Minn AJ, Gupta GP, Siegel PM, Bos PD, Shu WP, Giri DD, Viale A, Olshen AB, Gerald WL, Massague J: Genes that mediate breast cancer metastasis to lung. Nature 2005, 436:518-524.

20. Teschendorff AE, Naderi A, Barbosa-Morais NL, Pinder SE, Ellis IO, Aparicio S, Brenton JD, Caldas C: A consensus prognostic 
gene expression classifier for ER positive breast cancer. Genome Biol 2006, 7:R101.

21. Teschendorff AE, Miremadi A, Pinder S, Ellis I, Caldas C: An immune response gene expression module identifies a good prognosis subtype in estrogen receptor negative breast cancer. Genome Biol 2007, 8:R157.

22. Sotiriou $\mathrm{C}$, Piccart MJ: Taking gene-expression profiling to the clinic: when will molecular signatures become relevant to patient care? Nat Rev Cancer 2007, 7:545-553.

23. Ein-Dor L, Zuk O, Domany E: Thousands of samples are needed to generate a robust gene list for predicting outcome in cancer. Proc Natl Acad Sci USA 2006, 103:5923-5928.

24. Fan $C$, Oh DS, Wessels $L$, Weigelt $B$, Nuyten DS, Nobel AB, van't Veer LJ, Perou CM: Concordance among gene-expressionbased predictors for breast cancer. N Engl J Med 2006, 355:560-569.

25. Sotiriou C, Wirapati P, Loi S, Harris A, Fox S, Smeds J, Nordgren $\mathrm{H}$, Farmer P, Praz V, Haibe-Kains B, Desmedt C, Larsimont D, Cardoso F, Peterse H, Nuyten D, Buyse M, Vijver MJ van de, Bergh J, Piccart M, Delorenzi M: Gene expression profiling in breast cancer: understanding the molecular basis of histologic grade to improve prognosis. J Nat/ Cancer Inst 2006, 98:262-272.

26. Mook S, Schmidt MK, Viale G, Pruneri G, Eekhout I, Floore A, Glas AM, Bogaerts J, Cardoso F, Piccart-Gebhart MJ, Rutgers ET, Van't Veer LJ, On behalf of the TRANSBIG consortium: The 70-gene prognosis-signature predicts disease outcome in breast cancer patients with 1-3 positive lymph nodes in an independent validation study. Breast Cancer Res Treat 2008 in press.

27. Goldstein LJ, Gray R, Badve S, Childs BH, Yoshizawa C, Rowley S, Shak S, Baehner FL, Ravdin PM, Davidson NE, Sledge GW Jr, Perez EA, Shulman LN, Martino S, Sparano JA: Prognostic utility of the 21-gene assay in hormone receptor-positive operable breast cancer compared with classical clinicopathologic features. J Clin Oncol 2008, 26:4063-4071.

28. Pawitan Y, Bjöhle J, Amler L, Borg A-L, Egyhazi S, Hall P, Han X, Holmberg L, Huang F, Klaar S, Liu ET, Miller L, Nordgren H, Ploner A, Sandelin K, Shaw PM, Smeds J, Skoog L, Wedrén S, Bergh J: Gene expression profiling spares early breast cancer patients from adjuvant therapy: derived and validated in two population-based cohorts. Breast Cancer Res 2005, 7:R953-R964.

29. Chin K, DeVries S, Fridlyand J, Spellman PT, Roydasgupta R, Kuo W-L, Lapuk A, Neve RM, Qian Z, Ryder T: Genomic and transcriptional aberrations linked to breast cancer pathophysiologies. Cancer Cell 2006, 10:529-541.

30. Gene Expression Omnibus [http://www.ncbi.nlm.nih.gov/geo/]

31. ArrayExpress repository [http://www.ebi.ac.uk/microarray-as/ ae/]

32. $\mathbf{R}$ [http://cran.r-project.org/]

33. Bioconductor [http://www.bioconductor.org]

34. Matlab [http://www.mathworks.com/]

35. Gene Ontology [http://www.ncbi.nlm.nih.gov/]

36. Kyoto Encyclopedia of Genes and Genomes [http:// www.genome.ad.jp]

37. Reactome [http://www.reactome.org]

38. Molecular Signatures Database [http://www.broad.mit.edu/ gsea/]

39. Benjamini $Y$, Hochberg $Y$ : Controlling the false discovery rate: a practical and powerful approach to multiple testing. $J R$ Stat Soc Ser B 1995, 57:289-300.

40. Schemper M, Henderson R: Predictive accuracy and explained variation in Cox regression. Biometrics 2000, 56:249-255.

41. Lusa L, Miceli R, Mariani L: Estimation of predictive accuracy in survival analysis using $\mathbf{R}$ and S-PLUS. Comput Methods Programs Biomed 2007, 87:132-137.

42. Dunkler D, Michiels S, Schemper M: Gene expression profiling: does it add predictive accuracy to clinical characteristics in cancer prognosis? Eur J Cancer 2007, 43:745-751.

43. Buyse M, Loi $S$, van't Veer L, Viale $G$, Delorenzi M, Glas AM, d'Assignies MS, Bergh J, Lidereau R, Ellis P, Harris A, Bogaerts J, Therasse $P$, Floore A, Amakrane M, Piette F, Rutgers E, Sotiriou C, Cardoso F, Piccart MJ, the TRANSBIG Consortium: Validation and clinical utility of a 70-gene prognostic signature for women with node-negative breast cancer. J Natl Cancer Inst 2006, 98:1183-1192.

44. Whitfield ML, George LK, Grant GD, Perou CM: Common markers of proliferation. Nat Rev Cancer 2006, 6:99-106.
45. Chung $\mathrm{CH}$, Bernard PS, Perou CM: Molecular portraits and the family tree of cancer. Nat Genet 2002, 32(Suppl):533-540.

46. Yu J, Sieuwerts A, Zhang Y, Martens J, Smid M, Klijn J, Wang Y, Foekens J: Pathway analysis of gene signatures predicting metastasis of node-negative primary breast cancer. $B M C$ Cancer 2007, 7:182.

47. de Visser KE, Coussens LM: The inflammatory tumor microenvironment and its impact on cancer development. Contrib Microbio/ 2006, 13:118-137.

48. Galon J, Costes A, Sanchez-Cabo F, Kirilovsky A, Mlecnik B Lagorce-Pagès C, Tosolini M, Camus M, Berger A, Wind P, Zinzindohoué $F$, Bruneval $P$, Cugnenc $P-H$, Trajanoski $Z$, Fridman $W-H$, Pagès F: Type, density, and location of immune cells within human colorectal tumors predict clinical outcome. Science 2006, 313:1960-1964.

49. Venables JP: Aberrant and alternative splicing in cancer. Cancer Res 2004, 64:7647-7654.

50. Venables JP: Unbalanced alternative splicing and its significance in cancer. Bioessays 2006, 28:378-386.

51. Pajares MJ, Ezponda T, Catena R, Calvo A, Pio R, Montuenga LM: Alternative splicing: an emerging topic in molecular and clinical oncology. Lancet Oncol 2007, 8:349-357.

52. Mazoyer S, Puget N, Perrin-Vidoz L, Lynch HT, Serova-Sinilnikova OM, Lenoir GM: A BRCA1 nonsense mutation causes exon skipping. Am J Hum Genet 1998, 62:713-715.

53. Chen LL, Sabripour M, Wu EF, Prieto VG, Fuller GN, Frazier ML: A mutation-created novel intra-exonic pre-mRNA splice site causes constitutive activation of KIT in human gastrointestinal stromal tumors. Oncogene 2005, 24:4271-4280.

54. Watermann DO, Tang $Y$, Hausen $A Z$, Jäger M, Stamm S, Stickeler E: Splicing factor Tra2-beta1 is specifically induced in breast cancer and regulates alternative splicing of the CD44 gene. Cancer Res 2006, 66:4774-4780.

55. Hayes GM, Carrigan PE, Miller LJ: Serine-arginine protein kinase 1 overexpression is associated with tumorigenic imbalance in mitogen-activated protein kinase pathways in breast, colonic, and pancreatic carcinomas. Cancer Res 2007, 67:2072-2080.

56. Hayes GM, Carrigan PE, Beck AM, Miller LJ: Targeting the RNA splicing machinery as a novel treatment strategy for pancreatic carcinoma. Cancer Res 2006, 66:3819-3827.

57. Yang ZOS, Katie L, Ray ME, Abrams J, Ethier SP: Multiple interacting oncogenes on the 8p11-p12 amplicon in human breast cancer. Cancer Res 2006, 66:11632-11643.

58. Streicher KL, Yang ZQ, Draghici S, Ethier SP: Transforming function of the LSM1 oncogene in human breast cancers with the 8p11-12 amplicon. Oncogene 2007, 26:2104-2114.

59. van Vliet MH, Reyal F, Horlings HM, Vijver MJ van de, Reinders MJ, Wessels LF: Pooling breast cancer datasets has a synergetic effect on classification performance and improves signature stability. BMC Genomics 2008, 9:375. 